\title{
Sequential Probabilistic Ratio Test for the Scale Parameter of the $P$-Norm Distribution
}

\author{
Huan Ren $\mathbb{D}^{1},{ }^{1}$ Hongchang $\mathrm{Hu} \mathbb{D}^{\mathbb{D}},{ }^{1}$ and Zhen Zeng $\mathbb{D}^{2}$ \\ ${ }^{1}$ College of Mathematics and Statistics, Hubei Normal University, Huangshi 435002, China \\ ${ }^{2}$ School of Applied Mathematics, Nanjing University of Finance and Economics, Nanjing 210023, China \\ Correspondence should be addressed to Hongchang Hu; retutome@163.com
}

Received 1 April 2021; Revised 18 June 2021; Accepted 30 June 2021; Published 15 July 2021

Academic Editor: Shiping Wen

Copyright (c) 2021 Huan Ren et al. This is an open access article distributed under the Creative Commons Attribution License, which permits unrestricted use, distribution, and reproduction in any medium, provided the original work is properly cited.

We consider a series of independent observations from a $P$-norm distribution with the position parameter $\mu$ and the scale parameter $\sigma$. We test the simple hypothesis $H_{0}: \sigma=\sigma_{1}$ versus $H_{1}: \sigma=\sigma_{2}$. Firstly, we give the stop rule and decision rule of sequential probabilistic ratio test (SPRT). Secondly, we prove the existence of $h(\sigma)$ which needs to satisfy the specific situation in SPRT method, and the approximate formula of the mean sample function is derived. Finally, a simulation example is given. The simulation shows that the ratio of sample size required by SPRT and the classic Neyman-Pearson $(\mathrm{N}-\mathrm{P})$ test is about $50.92 \%$ at most, $38.30 \%$ at least.

\section{Introduction}

$P$-norm distribution is a family of distributions, including normal distribution, Laplace distribution, uniform distribution, degenerate distribution and many unknown distributions. Let the density function of the random variable be given by

$$
f(x)=\frac{p \lambda}{2 \sigma \Gamma(1 / p)} \exp \left\{-\left[\lambda \frac{|x-\mu|}{\sigma}\right]^{p}\right\} .
$$

Then, it is said that $X$ follows the $P$-norm distribution, where $\lambda=(\Gamma(3 / p) / \Gamma(1 / p))^{1 / 2}$, the position parameter is $\mu$, and the scale parameter is $\sigma>0$. Since it contains some important distributions, the $P$-norm distribution can better describe error distributions to some extent. There are many articles that studied the properties of the $P$-norm distribution. For example, Hu and Sun [1] systematically obtained unbiased estimators of parameters for the $P$-norm distribution. Sun and $\mathrm{Hu}$ [2] gave a density function of $P$-norm distribution and its sampling distribution. However, nobody investigated the sequential probabilistic ratio test for the scale parameter of $P$-norm distribution.

Likelihood ratio method is a widely used test method, which can get a lot of in-depth results. For example, Self and
Liang [3] gave the asymptotic distribution of maximum likelihood estimators and likelihood ratio statistics under nonstandard conditions. Fan and Zhang [4] proposed the sieve empirical likelihood ratio test for nonparametric functions. Ferrari and Cysneiros [5] used the Skovgaard's modified likelihood ratio method to study the exponential family nonlinear model and obtained the approximate distribution of modified likelihood ratio statistics. Giampaoli and Singer [6] tested the variance parameter of the linear mixed model by the likelihood ratio method. Huang et al. [7] tested the shape parameter of the generalized extremum distribution with the Lq-likelihood ratio method. Qin and Priebe [8] proposed a robust Lq-likelihood ratio test for the general pollution distribution and obtained the asymptotic distribution of Lq-likelihood ratio test statistics.

In order to meet the requirements of quality inspection of American munitions production during World War II, Wald [9] presented a sequential analysis method. Since then, many authors had studied sequence analysis methods and proposed various sequence tests to test different hypotheses. For example, Whitehead and Jones [10] and Jennison and Turnbull [11] provided extensive applications of sequence and group sequence based techniques in the formation and execution of clinical trials in their books. Darkhovsky [12] 
studied the sequence examination of two compound hypotheses and proposed a sequence process that minimizes the maximum Bayesian risk on a series of prior parameter distributions. Kachiashvili [13] proposed a sequential method to constrain the multiple test problem in the Bayesian hypothesis test task and proved the high quality of this method. Li et al. [14] extended the sequence probability ratio test and proved that the sequence test was asymptotically optimal when the error probability went to zero, so the sequence test could asymptotically obtain the minimum expected sample size. Wang et al. [15] proposed the weighted expected sample size (WESS) to evaluate the test problem of the composite hypothesis for the overall performance of three different regions. Nakamura et al. [16] proposed a sequential test procedure to determine the minimum dose with threshold effect. Li et al. [17] constructed a general sequence test to detect outliers in all collected observation sequences. Mudholkar et al. [18] deduced the sequential probabilistic ratio test method of M-Gaussian population model under the assumption that the discrete parameters were known. Zou et al. [19] proposed a nonparametric sequential test based on empirical likelihood to test the treatment effect. Tartakovsky et al. [20] discussed in detail recent advances in sequential hypothesis testing in their book.

The $P$-norm distribution describes the error distribution. In order to use the $P$-norm distribution, it is necessary to use the sequential likelihood ratio test method to study the $P$-norm distribution parameter. Therefore, this paper will apply the sequential probability ratio test method to study the parameter of $P$-norm distribution. The specific structure of this paper is as follows. Section 2 introduces the SPRT method. Section 3 gives some properties of SPRT. Section 4 performs a simulation study to confirm results. Proofs of theorems are contained in Section 5.

\section{SPRT Method}

Suppose that $\left(X_{1}, X_{2}, \ldots\right)$ is an independent and identically distributed (i.i.d.) random sample sequence from the $P$-norm distribution. Let $\left(x_{1}, x_{2}, \ldots\right)$ be the sequence of their observed values. The following is a simple hypothesis test problem (let us assume that parameter $\mu$ is known. Without loss of generality, let parameter $\mu=0$ ):

$$
H_{0}: \sigma=\sigma_{1} \leftrightarrow H_{1}: \sigma=\sigma_{2} .
$$

Here, $\sigma_{1}, \sigma_{2} \in \Theta$ are known numbers and $0<\sigma_{1}<\sigma_{2}$.

Let us give the stopping rule and decision rule of SPRT in turn. First, consider the likelihood ratio statistic:

$$
{ }_{\%}^{\lambda_{n}=} \frac{\prod_{i=1}^{n} f\left(X_{i}, \sigma_{2}\right)}{\prod_{i=1}^{n} f\left(X_{i}, \sigma_{1}\right)=\left(\frac{\sigma_{1}}{\sigma_{2}}\right)^{n} \exp \left\{\lambda^{p}\left(\sigma_{1}^{-p}-\sigma_{2}^{-p}\right) \sum_{i=1}^{n}\left|X_{i}\right|^{p}\right\} .}
$$

Let $S_{n}^{p}=\sum_{i=1}^{n}\left|X_{i}\right|^{p}$. Taking the logarithm of equation (3), we can get

$$
\ln \lambda_{n}=n \ln \frac{\sigma_{1}}{\sigma_{2}}+\lambda^{p}\left(\sigma_{1}^{-p}-\sigma_{2}^{-p}\right) S_{n}^{p} .
$$

Given the test level $\alpha>0$ and $\beta>0, \alpha+\beta<1$. Taking $A=$ $\beta /(1-\alpha)$ and $B=(1-\beta) / \alpha$, we can get

$$
\begin{aligned}
c & =-\frac{\ln \sigma_{1} / \sigma_{2}}{\lambda^{p}\left(\sigma_{1}^{-p}-\sigma_{2}^{-p}\right)}, \\
d_{1} & =\frac{\ln B}{\lambda^{p}\left(\sigma_{1}^{-p}-\sigma_{2}^{-p}\right)}, \\
d_{2} & =\frac{\ln A}{\lambda^{p}\left(\sigma_{1}^{-p}-\sigma_{2}^{-p}\right)} .
\end{aligned}
$$

We easily know $c>0, d_{1}>0$, and $d_{2}>0$. It is given by $\lambda_{n} \geq B$ that

$$
S_{n}^{p} \geq-\frac{\ln \sigma_{1} / \sigma_{2}}{\lambda^{p}\left(\sigma_{1}^{-p}-\sigma_{2}^{-p}\right)}+\frac{\ln B}{\lambda^{p}\left(\sigma_{1}^{-p}-\sigma_{2}^{-p}\right)}=c n+d_{1}=R_{n} .
$$

Similarly, it is given by $\lambda_{n} \geq A$ that

$$
S_{n}^{p} \leq-\frac{\ln \sigma_{1} / \sigma_{2}}{\lambda^{p}\left(\sigma_{1}^{-p}-\sigma_{2}^{-p}\right)}+\frac{\ln A}{\lambda^{p}\left(\sigma_{1}^{-p}-\sigma_{2}^{-p}\right)}=c n+d_{2}=A_{n} .
$$

It is not difficult to see that the sufficient and necessary condition for $\lambda_{n} \geq B$ is $S_{n}^{p} \geq R_{n}$, the sufficient and necessary condition of $\lambda_{n} \leq A$ is $S_{n}^{p} \leq A_{n}$. Then, the stopping rule of the SPRT for the $P$-norm distribution is

$$
\tau^{*}=\inf \left\{n: n \geq 1 \text { and } S_{n}^{p} \notin\left(A_{n}, R_{n}\right)\right\} .
$$

The decision rule of the $P$-norm distribution SPRT is as follows:

(i) When $S_{n}^{p} \geq R_{n}$, we should stop the experiment and accept $H_{0}$

(ii) When $S_{n}^{p} \leq A_{n}$, we should stop the experiment and reject $H_{0}$

\section{Sample Size Required for SPRT}

From the above analysis, we know that the SPRT method depends on the constants $A$ and $B$ that have been selected beforehand. For the selection of $A$ and $B$, we need to start from some properties of SPRT. The study of SPRT properties can be turned into the study of random walks. Then, this section will study the random walks of the SPRT method for the $P$-norm distribution and give some properties of SPRT.

Given $\alpha$ and $\beta$ (which satisfy $\alpha+\beta<1$ ), we can get constants $A$ and $B$. Let $z_{i}=\ln \left(f\left(X_{i}, \sigma_{2}\right) /\right.$ $\left.f\left(X_{i}, \sigma_{1}\right)\right)(i=1,2, \ldots)$. Then, the random walk sequence is $\left\{Z_{n}=\sum_{i=1}^{n} z_{i}, n \geq 1\right\}$. We have $L(\sigma)=P_{\sigma}\left(\right.$ accept $\left.H_{1}\right)$ $=P_{\sigma}\left(Z_{n} \leq \ln A\right)$ for $\forall \sigma \in \Theta$.

By (4), we can get 


$$
\begin{aligned}
Z_{1} & =\ln \frac{f\left(X_{1}, \sigma_{2}\right)}{f\left(X_{1}, \sigma_{1}\right)} \\
& =\ln \frac{\sigma_{1}}{\sigma_{2}} \exp \left(\lambda\left|X_{1}\right|\right)^{p}\left(\sigma_{1}^{-p}-\sigma_{2}^{-p}\right) \\
& =\ln \frac{\sigma_{1}}{\sigma_{2}}-\left(\lambda\left|X_{1}\right|\right)^{p}\left(\sigma_{2}^{-p}-\sigma_{1}^{-p}\right), \\
E Z_{1} & =E\left(\ln \frac{\sigma_{1}}{\sigma_{2}}-\left(\lambda\left|X_{1}\right|\right)^{p}\left(\sigma_{2}^{-p}-\sigma_{1}^{-p}\right)\right) \\
& =\ln \frac{\sigma_{1}}{\sigma_{2}}-\lambda^{p} E\left|X_{1}\right|^{p}\left(\sigma_{2}^{-p}-\sigma_{1}^{-p}\right) .
\end{aligned}
$$

Note that $E\left|X_{i}\right|^{p}=(1 / p)(\sigma / \lambda)^{p}$ and $E\left|X_{i}\right|^{2 p}=$ $\left((1+p) / p^{2}\right)(\sigma / \lambda)^{2 p}$ (Hu and Sun [1]). By (10), we have

$$
E Z_{1}=\ln \frac{\sigma_{1}}{\sigma_{2}}-\frac{\sigma^{p}}{p}\left(\sigma_{2}^{-p}-\sigma_{1}^{-p}\right) .
$$

If $E Z_{1} \geq 0$, then

$$
\sigma^{p} \geq \frac{p \ln \left(\sigma_{1} / \sigma_{2}\right)}{\sigma_{2}^{-p}-\sigma_{1}^{-p}}
$$

Let $\sigma_{*}^{p}=\left(p \ln \left(\sigma_{1} / \sigma_{2}\right)\right) /\left(\sigma_{2}^{-p}-\sigma_{1}^{-p}\right)$ we know that

(i) When $\sigma_{p}=\sigma_{*}^{p}, E Z_{1}=0$ can be given

(ii) When $\sigma_{p}>\sigma_{*}^{p}, E Z_{1}>0$ can be given

(iii) When $\sigma_{p}<\sigma_{*}^{p}, E Z_{1}<0$ can be given

Similarly, we have

$$
\begin{aligned}
Z_{1}^{2}= & \left(\ln \frac{\sigma_{1}}{\sigma_{2}}-\left[\lambda\left|X_{1}\right|\right]^{p}\left(\sigma_{2}^{-p}-\sigma_{1}^{-p}\right)\right)^{2} \\
= & \left(\ln \frac{\sigma_{1}}{\sigma_{2}}\right)^{2}+\lambda^{2 p}\left(\sigma_{2}^{-p}-\sigma_{1}^{-p}\right)^{2}\left|X_{1}\right|^{2 p} \\
& -2\left(\ln \frac{\sigma_{1}}{\sigma_{2}}\right) \lambda^{p}\left|X_{1}\right|^{p}\left(\sigma_{2}^{-p}-\sigma_{1}^{-p}\right), \\
E Z_{1}^{2}= & \left(\ln \frac{\sigma_{1}}{\sigma_{2}}\right)^{2}+\lambda^{2 p}\left(\sigma_{2}^{-p}-\sigma_{1}^{-p}\right)^{2}\left|X_{1}\right|^{2 p} \\
= & \left(\ln \frac{\sigma_{1}}{\sigma_{2}}\right)^{2}+\frac{p+1}{p}\left(\sigma_{2}^{-p}-\sigma_{1}^{-p}\right)^{2} \sigma^{2 p}-\frac{2}{p}\left(\ln \frac{\sigma_{1}}{\sigma_{2}}\right)\left(\sigma_{2}^{-p}-\sigma_{1}^{-p}\right) \sigma^{p} .
\end{aligned}
$$

For simplicity, $h=h(\sigma)$ is denoted as a function of $\sigma$.

$$
\begin{aligned}
\exp \left(h Z_{1}\right)= & \exp \left(h\left[\ln \frac{\sigma_{1}}{\sigma_{2}}-\left[\lambda\left|X_{1}\right|\right]^{p}\left(\sigma_{2}^{-p}-\sigma_{1}^{-p}\right)\right]\right), \\
E \exp \left(h Z_{1}\right)= & \exp \left(h \ln \frac{\sigma_{1}}{\sigma_{2}}\right) \frac{p \lambda}{2 \sigma \Gamma(1 / p)} \int_{-\infty}^{+\infty} \exp \\
& \left(-\lambda^{p}\left(h\left(\sigma_{2}^{-p}-\sigma_{1}^{-p}\right)+\sigma^{-p}\right)\left|X_{1}\right|^{p}\right) \mathrm{d} X_{1} .
\end{aligned}
$$

Let $h^{*}=\sigma^{-p} /\left(\sigma_{1}^{-p}-\sigma_{2}^{-p}\right), h^{*}>0$. We have

$E \exp \left(h Z_{1}\right)= \begin{cases}\frac{1}{\sigma} \exp \left(h \ln \frac{\sigma_{1}}{\sigma_{2}}\right)\left(h\left(\sigma_{2}^{-p}-\sigma_{1}^{-p}\right)+\sigma^{-p}\right)^{-1 / p}, & h<h^{*}, \\ \infty, & h \geq h^{*} .\end{cases}$

By studying random walks, we can derive some properties of SPRT. Next, we will give some properties of SPRT method for $P$-norm distributions. At the same time, we will also give the average sample size required for the SPRT method.

Theorem 1. If $h<\sigma^{-p} /\left(\sigma_{1}^{-p}-\sigma_{2}^{-p}\right)$ exists, then there must be some $h \neq 0$ that satisfies $E\left(\exp \left(h Z_{1}\right)\right)=1$.

In order to find out the average sample size in the SPRT method, we need to verify whether condition $h<\sigma^{-} P /\left(\sigma_{1}^{-p}-\right.$ $\left.\sigma_{2}^{-p}\right)$ in Theorem 1 is true. It is given by Theorem 2 .

Theorem 2. For $\sigma_{1}$ and $\sigma_{2}$ in (2), $h_{0}\left(\sigma_{i}\right)<\sigma_{i}^{-p} /\left(\sigma_{1}^{-p}-\sigma_{2}^{-p}\right)$ $(i=1,2)$.

The operational characteristic (OC) of Wald's SPRT is the probability of accepting the null hypothesis. The average sample number (ASN) is the average number of observations that we would have to collect in order to make a decision regarding the statistical hypotheses put forth. According to Mudholkar et al. [18], we can give the OC function and ANS of SPRT for the $P$-norm distribution. These are given by Theorems 3 and 4 .

Theorem 3. If $h<\sigma^{-p} /\left(\sigma_{1}^{-p}-\sigma_{2}^{-p}\right)$ and $E\left(\exp \left(h Z_{1}\right)\right)=1$, then the OC function of SPRT is given by

$$
L(\sigma)=P_{\sigma}\left(\operatorname{accept} H_{1}\right) \approx \frac{B^{h(\sigma)}}{B^{h(\sigma)}-A^{h(\sigma)}} .
$$

By Theorem 3, we can get the approximate formulas $\alpha \approx 1-L_{1}$ and $\beta \approx L_{2}$, where $L_{1}=L\left(\sigma_{1}\right)$ and $L_{2}=L\left(\sigma_{2}\right)$. Thus, we can give Theorem 4 .

Theorem 4. The ANS of SPRT for the P-norm distribution is that

(1) If $\sigma^{p}=\left(p \ln \left(\sigma_{1} / \sigma_{2}\right) /\left(\sigma_{2}^{-p}-\sigma_{1}^{-p}\right)\right)$, then $E_{\sigma} \tau^{*} \approx$ $\left(L(\sigma)(\ln A)^{2}+(1-L(\sigma))(\ln B)^{2}\right) / E_{\sigma} Z_{1}^{2}$

(2) If $\sigma^{p} \neq\left(p \ln \left(\sigma_{1} / \sigma_{2}\right) /\left(\sigma_{2}^{-p}-\sigma_{1}^{-p}\right)\right)$, then $E_{\sigma} \tau^{*} \approx$ ( $\left.(L(\sigma) \ln A+(1-L(\sigma)) \ln B) / E_{\sigma} Z_{1}\right)$

For the given probability $\alpha$ and $\beta$ which are the type 1 and the type 2 errors, $A=\beta /(1-\alpha)$ and $B=(1-\beta) / \alpha$ are taken, respectively. According to Theorem 4, it can be obtained that the average sample size of the SPRT method is

$$
E \tau^{*}=\frac{1}{2}\left(E_{\sigma_{1}} \tau^{*}+E_{\sigma_{2}} \tau^{*}\right)
$$

$E_{\sigma_{1}} \tau^{*}$ and $E_{\sigma_{2}} \tau^{*}$ here are determined in four cases as follows: 
(1) If $\sigma_{i}^{p}=\left(p \ln \left(\sigma_{1} / \sigma_{2}\right) /\left(\sigma_{2}^{-p}-\sigma_{1}^{-p}\right)\right), i=1,2$, then $E_{\sigma_{1}} \tau^{*} \approx \frac{(1-\alpha)(\ln \beta /(1-\alpha))^{2}+\alpha(\ln (1-\beta) / \alpha)^{2}}{E_{\sigma_{1}} Z_{1}^{2}}$, $E_{\sigma_{2}} \tau^{*} \approx \frac{\beta(\ln \beta /(1-\alpha))^{2}+(1-\beta)(\ln (1-\beta) / \alpha)^{2}}{E_{\sigma_{2}} Z_{1}^{2}}$,

where

$$
\begin{aligned}
E_{\sigma_{1}} Z_{1}^{2}= & \left(\ln \frac{\sigma_{1}}{\sigma_{2}}\right)^{2}+\frac{p+1}{p}\left(\sigma_{2}^{-p}-\sigma_{1}^{-p}\right)^{2} \sigma_{1}^{2 p} \\
& -\frac{2}{p}\left(\ln \frac{\sigma_{1}}{\sigma_{2}}\right)\left(\left(\frac{\sigma_{1}}{\sigma_{2}}\right)^{p}-1\right), \\
E_{\sigma_{2}} Z_{1}^{2}= & \left(\ln \frac{\sigma_{1}}{\sigma_{2}}\right)^{2}+\frac{p+1}{p}\left(\sigma_{2}^{-p}-\sigma_{1}^{-p}\right)^{2} \sigma_{2}^{2 p} \\
& -\frac{2}{p}\left(\ln \frac{\sigma_{1}}{\sigma_{2}}\right)\left(\left(1-\frac{\sigma_{2}}{\sigma_{1}}\right)^{p}\right) .
\end{aligned}
$$

(2) If $\sigma_{i}^{p} \neq\left(p \ln \left(\sigma_{1} / \sigma_{2}\right) /\left(\sigma_{2}^{-p}-\sigma_{1}^{-p}\right)\right), i=1,2$, then

$$
\begin{aligned}
& E_{\sigma_{1}} \tau^{*} \approx \frac{(1-\alpha) \ln (\beta /(1-\alpha))+\alpha \ln ((1-\beta) / \alpha)}{E_{\sigma_{1}} Z_{1}}, \\
& E_{\sigma_{2}} \tau^{*} \approx \frac{\beta \ln (\beta /(1-\alpha))+(1-\beta) \ln ((1-\beta) / \alpha)}{E_{\sigma_{2}} Z_{1}},
\end{aligned}
$$

where

$$
\begin{aligned}
& E_{\sigma_{1}} Z_{1}=\ln \frac{\sigma_{1}}{\sigma_{2}}-\frac{\left(\sigma_{1} / \sigma_{2}\right)^{p}-1}{p}, \\
& E_{\sigma_{2}} Z_{1}=\ln \frac{\sigma_{1}}{\sigma_{2}}-\frac{1-\left(\sigma_{2} / \sigma_{1}\right)^{p}}{p} .
\end{aligned}
$$

(3) If $\sigma_{1}^{p}=\left(p \ln \left(\sigma_{1} / \sigma_{2}\right) /\left(\sigma_{2}^{-p}-\sigma_{1}^{-p}\right)\right)$ $\sigma_{2}^{p} \neq\left(p \ln \left(\sigma_{1} / \sigma_{2}\right) /\left(\sigma_{2}^{-p}-\sigma_{1}^{-p}\right)\right)$, then

$$
\begin{aligned}
& E_{\sigma_{1}} \tau^{*} \approx \frac{(1-\alpha)(\ln \beta /(1-\alpha))^{2}+\alpha(\ln (1-\beta) / \alpha)^{2}}{E_{\sigma_{1}} Z_{1}^{2}}, \\
& E_{\sigma_{2}} \tau^{*} \approx \frac{\beta \ln \beta /(1-\alpha)+(1-\beta) \ln (1-\beta) / \alpha}{E_{\sigma_{2}} Z_{1}} .
\end{aligned}
$$

(4) If $\sigma_{1}^{p} \neq\left(p \ln \left(\sigma_{1} / \sigma_{2}\right) /\left(\sigma_{2}^{-p}-\sigma_{1}^{-p}\right)\right)$ and $\sigma_{2}^{p}=(p \ln$ $\left.\left(\sigma_{1} / \sigma_{2}\right) /\left(\sigma_{2}^{-p}-\sigma_{1}^{-p}\right)\right)$, then

$$
\begin{aligned}
& E_{\sigma_{1}} \tau^{*} \approx \frac{(1-\alpha) \ln \beta /(1-\alpha)+\alpha \ln (1-\beta) / \alpha}{E_{\sigma_{1}} Z_{1}}, \\
& E_{\sigma_{2}} \tau^{*} \approx \frac{\beta(\ln \beta /(1-\alpha))^{2}+(1-\beta)(\ln (1-\beta) / \alpha)^{2}}{E_{\sigma_{2}} Z_{1}^{2}} .
\end{aligned}
$$

To compare the methods of the simulation example in Section 4, we briefly introduce the Neyman-Pearson test method to calculate the average sample size. The principle of the $\mathrm{N}-\mathrm{P}$ test is to control the probability of making the first type of error within a given range and to find the test to make the probability of making the second type of error as small as possible, that is, to maximize the effectiveness of the test. Before proceeding to the results, we introduce these notions: $\left\{\left|X_{i}\right|^{p}\right\}(i=1,2, \ldots, n)$ is an independent sequence, and we take $S_{n}^{p}=\sum_{i=1}^{n}\left|X_{i}\right|^{p}$ and $\bar{X}^{p}=\left(S_{n}^{p} / n\right)$. Likewise, we know $E\left|X_{i}\right|^{p}=(1 / p)(\sigma / \lambda)^{p}$ and $D\left|X_{i}\right|^{p}=(1 / p)(\sigma / \lambda)^{2 p}$ (Hu and Sun [1]).

According to the Neyman-Pearson theory, the optimal fixed quantity is as follows. We should look for $n$ and $c$ that satisfy

$$
\begin{aligned}
& P_{\sigma_{1}}\left(\bar{X}^{p} \geq c\right)=\alpha, \\
& P_{\sigma_{2}}\left(\bar{X}^{p}<c\right)=\beta .
\end{aligned}
$$

By the central limit theorem, we know that $\bar{X}^{p}$ approximately follows a normal distribution $A N\left(E\left|X_{i}\right|^{p}\right.$, $\left.\left(D\left|X_{i}\right|^{P / n}\right)\right)$, namely,

$$
\frac{\sqrt{n}\left(\bar{X}^{p}-E\left|X_{i}\right|^{p}\right)}{\sqrt{D\left|X_{i}\right|^{p}}} \text { is } A N(0,1) \text {. }
$$

We can get

$$
\left\{\begin{array}{l}
P_{\sigma_{1}}\left(\frac{\sqrt{n}\left(\bar{X}^{p}-E_{\sigma_{1}}\left|X_{i}\right|^{p}\right)}{\sqrt{D_{\sigma_{1}}\left|X_{i}\right|^{p}}} \geq \frac{\sqrt{n}\left(c-E_{\sigma_{1}}\left|X_{i}\right|^{p}\right)}{\sqrt{D_{\sigma_{1}}\left|X_{i}\right|^{p}}}\right)=\alpha, \\
P_{\sigma_{2}}\left(\frac{\sqrt{n}\left(\bar{X}^{p}-E_{\sigma_{2}}\left|X_{i}\right|^{p}\right)}{\sqrt{D_{\sigma_{2}}\left|X_{i}\right|^{p}}}<\frac{\sqrt{n}\left(c-E_{\sigma_{2}}\left|X_{i}\right|^{p}\right)}{\sqrt{D_{\sigma_{2}}\left|X_{i}\right|^{p}}}\right)=\beta .
\end{array}\right.
$$

That is,

$$
\left\{\begin{array}{c}
K_{\alpha}=\frac{\sqrt{n}\left(c-E_{\sigma_{1}}\left|X_{i}\right|^{p}\right)}{\sqrt{D_{\sigma_{1}}\left|X_{i}\right|^{p}}} \\
K_{1-\beta}=\frac{\sqrt{n}\left(c-E_{\sigma_{2}}\left|X_{i}\right|^{p}\right)}{\sqrt{D_{\sigma_{2}}\left|X_{i}\right|^{p}}},
\end{array}\right.
$$

where $K_{\alpha}$ and $K_{1-\beta}$ represent quantiles of the standard normal distribution.

Then, we have

$$
\begin{aligned}
& n=\frac{K_{\alpha} \sqrt{D_{\sigma_{1}}\left|X_{i}\right|^{p}}}{\left(c-E_{\sigma_{1}}\left|X_{i}\right|^{p}\right)}, \\
& c=\frac{K_{\alpha} \sqrt{D_{\sigma_{1}}\left|X_{i}\right|^{p}} E_{\sigma_{1}}\left|X_{i}\right|^{p}-K_{1-\beta} \sqrt{D_{\sigma_{2}}\left|X_{i}\right|^{p}} E_{\sigma_{2}}\left|X_{i}\right|^{p}}{K_{\alpha} \sqrt{D_{\sigma_{1}}\left|X_{i}\right|^{p}}-K_{1-\beta} \sqrt{D_{\sigma_{2}}\left|X_{i}\right|^{p}}} .
\end{aligned}
$$


And the ratio of sample size required by SPRT and N - P test is that

$$
r=\frac{E_{\sigma_{1}} \tau^{*}+E_{\sigma_{2}} \tau^{*}}{2 n}
$$

\section{A Simulation Example}

In this section, we will calculate the average sample size obtained by the SPRT method and the Neyman-Pearson test method, respectively, and compare them to verify the superiority of the SPRT method.

Suppose that $\sigma_{1}=1, \sigma_{2}=2, p=1, \mu=0$, and $\alpha=\beta$ $=0.05$. The approximate values of the average sample size required by the SPRT method at the given test level are shown in Tables $1-3$.

In order to illustrate the advantages of the SPRT method, the sample size required by the Neyman-Pearson test at the given test level is presented here (see Table 4). Meanwhile, Table 5 shows the ratio of the sample size required by the SPRT method and Neyman-Pearson test method at the given test level.

From the above example, we can see that the ratio of sample size required by SPRT and N - P test is about $50.92 \%$ at most and $38.30 \%$ at least. It can be seen that the sample size required by the SPRT method is much less than the optimal sample size required by the Neyman-Pearson test method, which also shows the advantages of the SPRT method.

\section{Proof of SPRT Properties}

Proof. of Theorem 1. Suppose that there exists $h(\sigma) \neq 0$ such that $E\left(\exp \left(h Z_{1}\right)\right)=1$. Hence,

$$
\begin{gathered}
\frac{1}{\sigma} \exp \left(h \ln \frac{\sigma_{1}}{\sigma_{2}}\right)\left(h\left(\sigma_{2}^{-p}-\sigma_{1}^{-p}\right)+\sigma^{-p}\right)^{-(1 / p)}=1, \\
\exp \left(h \ln \frac{\sigma_{1}}{\sigma_{2}}\right)-\sigma\left(h\left(\sigma_{2}^{-p}-\sigma_{1}^{-p}\right)+\sigma^{-p}\right)^{(1 / p)}=0 .
\end{gathered}
$$

Here, let $g(h)=\exp \left(h \ln \left(\sigma_{1} / \sigma_{2}\right)\right)-\sigma\left(h\left(\sigma_{2}^{-p}-\sigma_{1}^{-p}\right)+\right.$ $\left.\sigma^{-p}\right)^{(1 / p)}$. By calculation, we know $g(0)=0, g(-\infty)>0$, and $g\left(h^{*}\right)=\exp \left(h^{*} \ln \left(\sigma_{1} / \sigma_{2}\right)\right)>0$. To get $g(h)=0$, there exists $h$ such that $g(h)<0$, namely,

$$
\begin{aligned}
& \exp \left(h \ln \frac{\sigma_{1}}{\sigma_{2}}\right)-\sigma\left(h\left(\sigma_{2}^{-p}-\sigma_{1}^{-p}\right)+\sigma^{-p}\right)^{(1 / p)}<0, \\
& \left(\frac{\sigma_{1}}{\sigma_{2}}\right)^{h p}<h \sigma^{p}\left(\sigma_{2}^{-p}-\sigma_{1}^{-p}\right)+1 .
\end{aligned}
$$

Let $f(h)=\left(\sigma_{1} / \sigma_{2}\right)^{h p}-h \sigma^{p}\left(\sigma_{2}^{-p}-\sigma_{1}^{-p}\right)-1$; we have

$$
\begin{aligned}
& f^{\prime}(h)=p\left(\frac{\sigma_{1}}{\sigma_{2}}\right)^{h p} \ln \frac{\sigma_{1}}{\sigma_{2}}-\sigma^{p}\left(\sigma_{2}^{-p}-\sigma_{1}^{-p}\right), \\
& f^{\prime \prime}(h)=\left(p \ln \frac{\sigma_{1}}{\sigma_{2}}\right)^{2}\left(\frac{\sigma_{1}}{\sigma_{2}}\right)^{h p}>0 .
\end{aligned}
$$

TABLE 1: $E_{\sigma_{1}} \tau^{*}$ approximation.

\begin{tabular}{lcccc}
\hline$\beta$ & \multicolumn{3}{c}{$\alpha$} \\
& 0.01 & 0.03 & 0.05 & 0.1 \\
\hline 0.01 & 23.3150 & 22.4315 & 21.6255 & 19.7806 \\
0.03 & 17.6849 & 16.9173 & 16.2272 & 14.6720 \\
0.05 & 15.0677 & 14.3552 & 13.7201 & 12.3026 \\
0.1 & 11.5177 & 10.8825 & 10.3248 & 9.1007 \\
\hline
\end{tabular}

TABLE 2: $E_{\sigma_{2}} \tau^{*}$ approximation.

\begin{tabular}{lcccc}
\hline$\beta$ & \multicolumn{5}{c}{$\alpha$} \\
& 0.01 & 0.03 & 0.05 & 0.1 \\
\hline 0.01 & 14.6755 & 11.1317 & 9.4842 & 7.2498 \\
0.03 & 14.1194 & 10.6485 & 9.0358 & 6.8499 \\
0.05 & 13.6121 & 10.2141 & 8.6360 & 6.4989 \\
0.1 & 12.4508 & 9.2353 & 7.7438 & 5.7284 \\
\hline
\end{tabular}

TABLE 3: $E \tau^{*}$ approximation.

\begin{tabular}{lcccc}
\hline$\beta$ & \multicolumn{3}{c}{$\alpha$} \\
& 0.01 & 0.03 & 0.05 & 0.1 \\
\hline 0.01 & 18.9952 & 16.7816 & 15.5549 & 13.5152 \\
0.03 & 15.9022 & 13.7829 & 12.6315 & 10.7610 \\
0.05 & 14.3399 & 12.2846 & 11.1781 & 9.4007 \\
0.1 & 11.9843 & 10.0589 & 9.0343 & 7.4146 \\
\hline
\end{tabular}

So, we know that $f(h)$ is convex. Let us set $f^{\prime}(h)=0$ here, and we get

$$
h_{0}=\frac{\ln \left(\sigma^{p}\left(\sigma_{2}^{-p}-\sigma_{1}^{-p}\right) / p \ln \left(\sigma_{1} / \sigma_{2}\right)\right)}{p \ln \left(\sigma_{1} / \sigma_{2}\right)} .
$$

By calculation, we can know $h_{0} \neq 0$. We substitute $h_{0}$ into $f(h)$ and talk about the following two cases:

(1) If $\sigma=\sigma_{1}$, then

$$
f\left(h_{0}\right)=\frac{\sigma_{1}^{p} \sigma_{2}^{-p}-1}{p \ln \left(\sigma_{1} / \sigma_{2}\right)}\left(1-\ln \left(\frac{\sigma_{1}^{p} \sigma_{2}^{-p}-1}{p \ln \left(\sigma_{1} / \sigma_{2}\right)}\right)\right)-1 .
$$

Here, we study the properties of the function $V(t)=t(1-\ln t)-1$, where $t>0$. After calculation, we can know that the function value of $V(t)$ in its domain is always less than 0 . And $f\left(h_{0}\right)<0$ is always true if $\left(\sigma_{1}^{p} \sigma_{2}^{-p}-1\right) / p \ln \left(\sigma_{1} / \sigma_{2}\right)$ is regarded as a value of the independent variable in (35), where $\left(\left(\sigma_{1}^{p} \sigma_{2}^{-p}-1\right) / p \ln \left(\sigma_{1} / \sigma_{2}\right)\right)>0$. Thus, we can find $h_{0}$ to make the inequality (32) true, and then there is $h$ to make $g(h)<0$.

(2) If $\sigma=\sigma_{2}$, then

$$
f\left(h_{0}\right)=\frac{1-\sigma_{1}^{-p} \sigma_{2}^{p}}{p \ln \left(\sigma_{1} / \sigma_{2}\right)}\left(1-\ln \left(\frac{1-\sigma_{1}^{-p} \sigma_{2}^{p}}{p \ln \left(\sigma_{1} / \sigma_{2}\right)}\right)\right)-1 .
$$


TABle 4: Sample size required for the $\mathrm{N}-\mathrm{P}$ test method.

\begin{tabular}{lcccc}
\hline$\beta$ & \multicolumn{3}{c}{$\alpha$} \\
& 0.01 & 0.03 & 0.05 & 0.1 \\
\hline 0.01 & 48.8601 & 42.7716 & 39.8161 & 35.2836 \\
0.03 & 37.0881 & 31.8096 & 29.2681 & 25.4016 \\
0.05 & 31.6969 & 26.8324 & 24.5025 & 20.9764 \\
0.1 & 23.9121 & 19.7136 & 17.7456 & 14.7456 \\
\hline
\end{tabular}

TABLE 5: Ratio of sample size required for the SPRT and N - P test (\%).

\begin{tabular}{lcccc}
\hline$\beta$ & \multicolumn{5}{c}{$\alpha$} \\
& 0.01 & 0.03 & 0.05 & 0.1 \\
\hline 0.01 & 0.3888 & 0.3924 & 0.3907 & 0.3830 \\
0.03 & 0.4288 & 0.4333 & 0.4316 & 0.4236 \\
0.05 & 0.4524 & 0.4578 & 0.4562 & 0.4482 \\
0.1 & 0.5012 & 0.5103 & 0.5092 & 0.5028 \\
\hline
\end{tabular}

And by the same token, we get $f\left(h_{0}\right)<0$ here, we have the situation where $h$ makes $g(h)<0$.

To sum up, when $h(\sigma)<\left(\sigma^{-p} /\left(\sigma_{1}^{-p}-\sigma_{2}^{-p}\right)\right)$, it can be proved that $h(\sigma) \neq 0$ satisfies $E\left(\exp \left(h Z_{1}\right)\right)=1$ by the intermediate value theorem. In the end, the proposition has been proved.

Proof. of Theorem 2. When $\sigma=\sigma_{1}$, we can get

$$
h_{0}\left(\sigma_{1}\right)<\frac{\sigma_{1}^{-p}}{\sigma_{1}^{-p}-\sigma_{2}^{-p}}
$$

that is,

$$
\frac{\ln \left(\left(\left(\sigma_{1} / \sigma_{2}\right)^{p}-1\right) / p \ln \left(\sigma_{1} / \sigma_{2}\right)\right)}{p \ln \left(\sigma_{1} / \sigma_{2}\right)}<\frac{1}{1-\left(\sigma_{1} / \sigma_{2}\right)^{p}} .
$$

By observing the right part of (38), we know that its range is $(1,+\infty)$. Then, formula (38) can also be written as

$$
\frac{\ln \left(\left(\left(\sigma_{1} / \sigma_{2}\right)^{p}-1\right) /\left(p \ln \left(\sigma_{1} / \sigma_{2}\right)\right)\right)}{p \ln \left(\sigma_{1} / \sigma_{2}\right)} \leq 1 .
$$

By simplifying (39), it can be obtained that

$$
\begin{aligned}
\ln \left(\frac{\left(\sigma_{1} / \sigma_{2}\right)^{p}-1}{p \ln \left(\sigma_{1} / \sigma_{2}\right)}\right) & \geq p \ln \frac{\sigma_{1}}{\sigma_{2}}, \\
\left(\frac{\sigma_{1}}{\sigma_{2}}\right)^{p}-1 & \leq p\left(\frac{\sigma_{1}}{\sigma_{2}}\right)^{p} \ln \frac{\sigma_{1}}{\sigma_{2}} .
\end{aligned}
$$

Let $x=\left(\sigma_{1} / \sigma_{2}\right), x \in(0,1)$, and $T(x)=x^{p}-p x^{p} \ln x-$ 1 ; then,

$$
\begin{aligned}
T^{\prime}(x) & =p x^{p-1}-p x^{p-1}-p^{2} x^{p-1} \ln x \\
& =-p^{2} x^{p-1} \ln x>0 .
\end{aligned}
$$

From the above, we can see that the function $T(x)$ increases monotonically on $(0,1)$, and $T(1)=0$; hence, $T(x) \leq 0$ holds on $(0,1)$. And we can deduce that $\left(\sigma_{1} / \sigma_{2}\right)^{p}-1 \leq p\left(\sigma_{1} / \sigma_{2}\right)^{p} \ln \left(\sigma_{1} / \sigma_{2}\right) ;$ therefore, $h_{0}\left(\sigma_{1}\right)<\left(\sigma_{1}^{-p}\right.$ $\left./\left(\sigma_{1}^{-p}-\sigma_{2}^{-p}\right)\right)$ holds.

Similarly, when $\sigma=\sigma_{2}$, the inequality $h_{0}\left(\sigma_{2}\right)<$ $\left(\sigma_{2}^{-p} /\left(\sigma_{1}^{-p}-\sigma_{2}^{-p}\right)\right)$ also holds. To sum up, the proposition is proved.

\section{Data Availability}

No data were used to support this study.

\section{Conflicts of Interest}

The authors declare that there are no conflicts of interest.

\section{Acknowledgments}

This work was supported by the Natural Science Foundation of China (Grant no. 11471105).

\section{References}

[1] H. C. Hu and H. Y. Sun, "Parameters $\sigma$ estimation of $P$-norm distribution," Geomatics and Information Science of Wuhan University, vol. 27, no. 5, pp. 483-485, 2002.

[2] H. Y. Sun and H. C. Hu, "P-norm distribution and its sampling distribution," Chinese Journal of Applied Probability and Statistics, vol. 19, no. 4, pp. 424-428, 2003.

[3] S. G. Self and K. Y. Liang, "Asymptotic properties of maximum likelihood estimators and likelihood ratio tests under nonstandard conditions," Journal of the American Statistical Association, vol. 82, no. 398, pp. 605-610, 1987.

[4] J. Q. Fan and J. Zhang, "Sieve empirical likelihood ratio tests for nonparametric functions," The Annals of Statistics, vol. 32, no. 5, pp. 1858-1907, 2004.

[5] S. L. P. Ferrari and A. H. M. A. Cysneiros, "Skovgaard's adjustment to likelihood ratio tests in exponential family nonlinear models," Statistics \& Probability Letters, vol. 78, no. 17, pp. 3047-3055, 2008.

[6] V. Giampaoli and J. M. Singer, "Likelihood ratio tests for variance components in linear mixed models," Journal of Statistical Planning and Inference, vol. 139, no. 4, pp. 14351448, 2009.

[7] C. Huang, J. G. Lin, and Y. Y. Ren, "Testing for the shape parameter of generalized extreme value distribution based on the $L_{q}$ likelihood ratio statistic," Metrika, vol. 76, no. 5, pp. 641-671, 2013.

[8] Y. Qin and C. E. Priebe, "Robust hypothesis testing via LqLikelihood," Statistica Sinica, vol. 27, no. 4, pp. 1793-1813, 2017.

[9] A. Wald, Sequential Analysis, Wiley, New York, NY, USA, 1947.

[10] J. Whitehead and D. R. Jones, The Design and Analysis of Sequential Clinical Trials, Wiley, New York, NY, USA, 1983.

[11] C. Jennison and B. W. Turnbull, Group Sequential Methods with Applications to Clinical Trials, Chapman and Hall/CRC, Boca Raton, FL, USA, 1999.

[12] B. Darkhovsky, "Optimal sequential tests for testing two composite and multiple simple hypotheses," Sequential Analysis, vol. 30, no. 4, pp. 479-496, 2011.

[13] K. J. Kachiashvili, "The methods of sequential analysis of bayesian type for the multiple testing problem," Sequential Analysis, vol. 33, no. 1, pp. 23-38, 2014. 
[14] X. O. Li, J. C. Liu, and Z. L. Ying, "Generalized sequential probability ratio test for separate families of hypotheses," Sequential Analysis, vol. 33, no. 4, pp. 539-563, 2014.

[15] L. Wang, X. L. Pu, and Y. Li, "Asymptotic optimality of combined double sequential weighted probability ratio test for three composite hypotheses," Mathematical Problems in Engineering, vol. 2015, no. 6, 8 pages, 2015.

[16] T. Nakamura, Y. Yamamoto, and H. Douke, "Sequential hypothesis tests for identifying the minimum dose with a threshold effect," Communications in Statistics-Simulation and Computation, vol. 45, no. 6, pp. 1950-1970, 2016.

[17] Y. Li, S. Nitinawarat, and V. V. Veeravalli, "Universal sequential outlier hypothesis testing," Sequential Analysis, vol. 36, no. 3, pp. 309-344, 2017.

[18] G. S. Mudholkar, Z. Yu, and S. S. Awadalla, "Sequential probability ratio test for the mode of $M$-Gaussian distribution," Sequential Analysis, vol. 35, no. 2, pp. 226-237, 2016.

[19] L. Zou, A. Vexler, J. Yu, and H. Wan, "A sequential densitybased empirical likelihood ratio test for treatment effects," Statistics in Medicine, vol. 38, no. 12, pp. 2115-2125, 2019.

[20] A. Tartakovsky, I. Nikiforov, and M. Basseville, Sequential Analysis: Hypothesis Testing and Changepoint Detection, Chapman and Hall/CRC, Boca Raton, FL, USA, 2015. 